\title{
Loss of cysteinyl-tRNA synthetase (CARS) induces the transsulfuration pathway and inhibits ferroptosis induced by cystine deprivation
}

\author{
M Hayano', WS Yang ${ }^{2}$, CK Corn ${ }^{2}$, NC Pagano ${ }^{2}$ and BR Stockwell ${ }^{\star x, 2,3,4}$
}

Ferroptosis is a form of regulated non-apoptotic cell death that has been implicated in several disease contexts. A better understanding of the ferroptotic death mechanism could lead to the development of new therapeutics for degenerative diseases, and a better understanding of how to induce ferroptosis in specific tumor contexts. We performed an unbiased genome-wide siRNA screen to find genetic suppressors of ferroptosis. We determined that loss of CARS, the cysteinyl-tRNA synthetase, suppresses ferroptosis induced by erastin, which inhibits the cystine-glutamate antiporter known as system $\mathrm{x}_{\mathrm{c}}^{-}$. Knockdown of CARS inhibited erastin-induced death by preventing the induction of lipid reactive oxygen species, without altering iron homeostasis. Knockdown of CARS led to the accumulation of cystathionine, a metabolite on the transsulfuration pathway, and upregulated genes associated with serine biosynthesis and transsulfuration. In addition, inhibition of the transsulfuration pathway resensitized cells to erastin, even after CARS knockdown. These studies demonstrate a new mechanism of resistance to ferroptosis and may lead to strategies for inducing and suppressing ferroptosis in diverse contexts.

Cell Death and Differentiation (2016) 23, 270-278; doi:10.1038/cdd.2015.93; published online 17 July 2015

Precise regulation of cell death is essential for tissue homeostasis. Dysregulation of cell death processes is implicated in a variety of pathological conditions, such as ischemia and neurodegenerative diseases, providing a rationale for exploring cell-death-modulating compounds as potential therapeutics. ${ }^{1}$ However, an incomplete understanding of cell death mechanisms in specific disease contexts has hindered efforts to develop therapeutics. Mechanistic analyses of cell death processes in disease contexts may uncover new strategies for drug discovery. Ferroptosis, a form of oxidative, non-apoptotic cell death, has recently been described and implicated in several pathological conditions, including Huntington's disease $(H D)$, periventricular leukomalacia (PVL) and kidney dysfunction. ${ }^{2-4}$ Ferroptotic cell death can be induced through perturbation of redox homeostasis maintained by glutathione, a key regulator of the intracellular redox state.

Glutathione (GSH) is a tripeptide, the synthesis of which is dependent on the availability of the amino acid cysteine. A substantial fraction of extracellular cysteine exists as its oxidized disulfide form, cystine, because of the oxidative extracellular environment. ${ }^{5}$ Some cells primarily obtain cysteine by importing extracellular cystine through system $\mathrm{x}_{\mathrm{c}}^{-}$, the cystine-glutamate antiporter. Cystine is then reduced to cysteine inside cells, fueling GSH synthesis. GSH maintains redox homeostasis by acting as a reductive substrate for reactive oxygen species (ROS)-detoxifying enzymes. As one example, glutathione peroxidase 4 (GPX4) uses GSH to reduce lipid hydroperoxides and organic hydroperoxides to alcohols, serving a critical role in lipid repair and detoxification. GPX4 was recently shown to be a central regulator of ferroptosis. ${ }^{6}$

Ferroptosis can be induced by two classes of compounds, exemplified by erastin and $(1 S, 3 R)-$ RSL3. $^{6-9}$ These two compounds target different parts of the ferroptotic pathway. Erastin inhibits system $x_{c}^{-}$to deplete GSH, which effectively inactivates all cellular glutathione peroxidases, including GPX4. RSL3, on the other hand, acts downstream, inhibiting GPX4 directly. In both cases, the loss of GPX4 activity causes accumulation of lipid peroxides, and ultimately, cell death. Recently, the FDA-approved drugs sorafenib and sulfasalazine were also found to induce ferroptosis through inhibition of system $X_{c}^{-}$activity, ${ }^{10,11}$ although these lower-potency compounds may also activate other competing processes at similar or slightly higher concentrations. A specific inhibitor of ferroptosis, ferrostatin-1, and its analogs have been shown to suppress cell death in several degenerative disease models, including HD, PVL and kidney dysfunction, as well as in a model of glutamate toxicity, suggesting the involvement of ferroptosis in these conditions. ${ }^{4,12}$ Collectively, these findings

${ }^{1}$ Department of Pharmacology, Columbia University, 550 West 120th Street, Northwest Corner Building, MC 4846, New York, NY 10027, USA; ${ }^{2}$ Department of Biological Sciences, Columbia University, 550 West 120th Street, Northwest Corner Building, MC 4846, New York, NY 10027, USA; ${ }^{3}$ Department of Chemistry, Columbia University, 550 West 120th Street, Northwest Corner Building, MC 4846, New York, NY 10027, USA and ${ }^{4}$ Howard Hughes Medical Institute, Columbia University, 550 West 120 th Street, Northwest Corner Building, MC 4846, New York, NY 10027, USA

*Corresponding author: BR Stockwell, Department of Biological Sciences, Columbia University, 550 West 120th Street, Northwest Corner Building, MC 4846, New York, NY 10027, USA. Tel: +212 854 2899; Fax: +212 854 3293; E-mail: bstockwell@columbia.edu

Abbreviations: HD, Huntington's disease; PVL, periventricular leukomalacia; GSH, glutathione; ROS, reactive oxygen species; GPX4, glutathione peroxidase 4; BSO, bluthionine sulfoximine; CARS, cysteinyl-tRNA synthetase; CSSG, cysteine-glutathione disulfide; PPG, propargylglycine; ATOC, $\alpha$-tocopherol; DFOM, deferoxamine; CBS, cystathionine $\beta$-synthase

Received 06.1.15; revised 21.5.15; accepted 03.6.15; Edited by P Vendenabeele; published online 17.7.15 
suggest that modulation of ferroptosis is of potential therapeutic relevance in several pathological conditions.

Given the involvement of ferroptosis in these different contexts, we sought to identify specific features and regulators of ferroptosis. Ferroptosis is biochemically and morphologically distinct from necrosis and apoptosis. ${ }^{12}$ Genetic analysis of ferroptosis has been performed using a limited set of genes related to mitochondrial function. ${ }^{12}$ This previous analysis revealed that ferroptosis requires a distinct set of genes compared with apoptosis. However, this analysis cast a relatively narrow net; therefore, we sought to extend our understanding of the genetic regulation of ferroptosis further to identify essential genes and pathways using a genome-wide siRNA screen. Such genes may illuminate novel targets whose inhibition could be therapeutic in disease conditions involving aberrant activation of ferroptosis, or suggest strategies for inducing ferroptosis in specific tumor contexts.

\section{Results}

RNAi screen to identify genes essential for ferroptosis. In order to identify genes essential for ferroptosis in an unbiased fashion, we performed a screen in HT-1080 fibrosarcoma cells using a siRNA library targeting 21687 human genes (Ambion Silencer, Grand Island, NY, USA). A pool of three siRNA sequences targeting each gene was tested in 384-well format to identify suppressors of erastininduced ferroptosis. A pool of siRNAs targeting VDAC3, which was previously identified as an essential gene for erastin-induced ferroptosis, was used as a positive control on each plate (Figure 1a). ${ }^{9}$ HT-1080 cells were reverse transfected with siRNAs for $72 \mathrm{~h}$, after which they were treated with either $7 \mu \mathrm{M}$ erastin $\left(\mathrm{Gl}_{100}\right.$, the concentration required for $100 \%$ growth inhibition; this meant that we could only detect suppressors, not enhancers, in the screen) or an equivalent volume of DMSO as a vehicle control, for $24 \mathrm{~h}$ (Figure 1b). Growth inhibition (\%Gl) was calculated for each pool of siRNAs, and screening data were analyzed using CellHTS2, an R-based open source software package. ${ }^{13}$ Gene knockdowns that prevented erastin-induced ferroptosis by more than three median absolute distribution (MAD) from the median were rescreened in a second cell line (BJeLR), in the same manner as in HT-1080 cells (Supplementary Figure S1A). ${ }^{8}$ BJeLR cells are fibroblast cells harboring oncogenic hTERT, Simian Virus 40 large and small T oncogenes and oncogenic HRAS, and are sensitive to ferroptosis. ${ }^{14}$

A total of 1426 siRNA pools were retested in BJeLR cells; 325 showed $>50 \%$ rescue of erastin-induced ferroptosis in this cell line. siRNA pools targeting 250 genes were cherry picked based on the magnitude of rescue from erastin-induced ferroptosis, excluding genes that were independently toxic ( $<10 \%$ remaining viability without erastin treatment). siRNAs targeting the 250 selected genes were retested in a deconvoluted format (three independent siRNAs against each gene tested in separate wells) in a third cell line, Panc-1 (pancreatic epithelial carcinoma), as well as again in HT-1080 cells. We used Panc-1 cells to decrease the chances of finding a genetic modulator of ferroptosis that might be cell-line or lineage specific, and because Panc- 1 cells are derived from a different tissue lineage, but are sensitive to ferroptosis. Toxic siRNAs that decreased viability to $<30 \%$ of control were not further evaluated. Eleven genes reproducibly suppressed erastin-induced ferroptosis upon knockdown in both HT-1080 cells (three sequences, $t$-test, $P<0.05$ compared with nontargeting siRNA) and Panc- 1 cells (three sequences, $t$-test, $P<0.05$; Supplementary Table SI1). These 11 genes were associated with distinct pathways. Knockdown of CARS had by far the most robust suppressive effect, so we further investigated the function of this gene in ferroptosis (Figure 1c).

CARS knockdown inhibits erastin-induced ferroptosis in diverse cell contexts. CARS encodes the cysteinyl-tRNA synthetase, an enzyme involved in charging of tRNAs with cysteine for protein translation. ${ }^{15}$ We first validated the ability of CARS knockdown to prevent erastin-induced ferroptosis using three additional sequences of Ambion Silencer Select siRNAs targeting CARS (Figure 2a). These siRNA sequences knocked down CARS protein and mRNA levels as determined through immunoblotting and qPCR (Figure 2b). Independent shRNA constructs targeting CARS also showed a suppressive phenotype, corresponding to the degree of mRNA knockdown (Supplementary Figure S1B). CARS knockdown with siRNA was able to rescue from erastin treatment for longer than $24 \mathrm{~h}$ (Supplementary Figure S1C). Moreover, overexpression of a CARS cDNA construct, with the third codon of the siRNA target sequence mutated, prevented knockdown of CARS with the siRNAs and reversed the suppressive effects of siCARS, restoring sensitivity to erastin (Supplementary Figure S2A). CARS overexpression on its own did not sensitize to erastin-induced lethality, suggesting that excess CARS abundance does not further contribute to erastin-induced ferroptosis (Supplementary Figure $\mathrm{S} 2 \mathrm{~B}$ ). These experiments established that CARS expression is essential for erastin-mediated ferroptosis.

Erastin is lethal to many cell lines. ${ }^{12}$ To ensure that the suppressive effect of CARS knockdown was not specific to HT-1080, Panc-1 and BJeLR cells, we knocked down CARS in two additional erastin-sensitive cell lines-A673 (Ewing's sarcoma) and 143B (osteosarcoma) cells. CARS knockdown indeed prevented erastin-induced ferroptosis in all five of these cell lines, providing further evidence that CARS is required for ferroptosis in diverse cell contexts (Figure $2 \mathrm{c}$ and Supplementary Figure S2C).

We evaluated the specificity of the suppressive effect of CARS knockdown on lethal insults other than erastin. Specifically, we tested two other compounds that induce ferroptosis through distinct mechanisms (1 S,3R)-RSL3 (hereafter referred to as RSL3, which inhibits GPX4 directly) and FIN56, which causes depletion of GPX4 protein (Shimada et al., under review), and on the lethality of the apoptosis inducers doxorubicin (a DNA-damaging agent and topoisomeriase inhibitor) and rotenone (an inducer of mitochondrial ROS generation), in HT-1080 cells. CARS knockdown did not suppress the lethality of any of these compounds (Figure 2d), indicating that the suppressive effect of $C A R S$ is likely specific for ferroptosis induced by system $\mathrm{X}_{\mathrm{c}}^{-}$inhibition. In support of this observation, the lethality of ME II and PE, two erastin analogs that also inhibit system $\mathrm{x}_{\mathrm{c}}^{-}$, was suppressed by $C A R S$ knockdown. Given that RSL3 and FIN56 act at the level of 
a

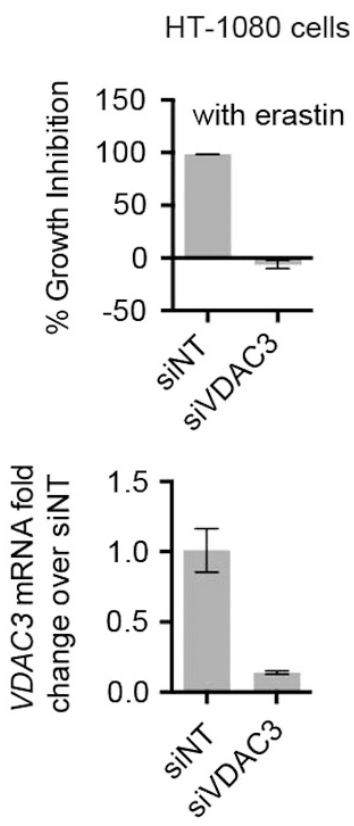

b

Genome-wide Screening Cascade 21,687 genes

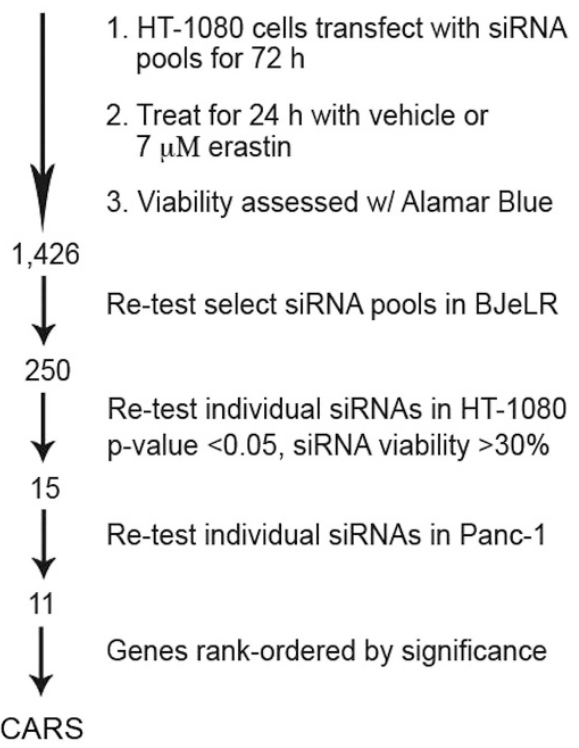

C Re-testing of the top 250 siRnA pools in HT-1080

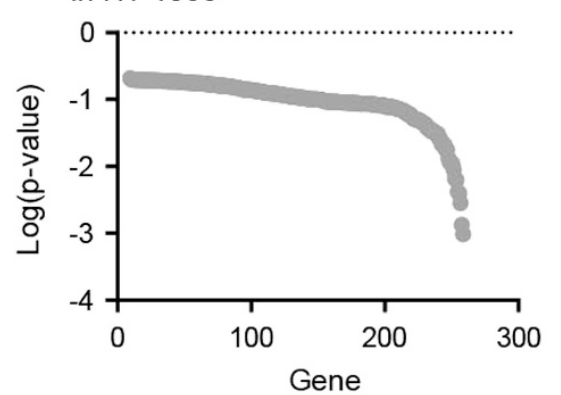

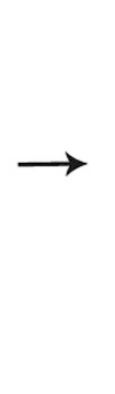

Hit-selection

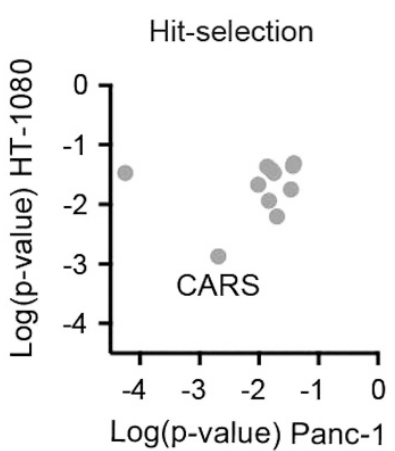

Figure 1 Genome-wide RNAi screen for inhibitors of erastin-induced ferroptosis. (a) siRNA targeting VDAC3 was used in HT-1080 fibrosarcoma cells. $10 \mathrm{nM}$ siRNA was reverse transfected into cells in 384-well format. qPCR was used to verify knockdown in six-well plates. Error bars represent one S.D. using three biological replicates. (b) Screening format used to identify suppressors of erastin lethality. (c) Effect of 250 gene knockdowns on erastin lethality, ordered in rank of most significant in HT-1080 cells. On the right, gene knockdowns were filtered for lack of toxicity and $t$-test $P$-value $<0.05$, in both $\mathrm{HT}-1080$ and Panc- 1 cells

GPX4 inactivation, leading to accumulation of lipid peroxides and execution of ferroptosis, ${ }^{6}$ we hypothesized that $C A R S$ knockdown inhibits erastin's lethality upstream of GPX4. CARS knockdown did not suppress the lethality of buthionine sulfoximine (BSO), an inhibitor of $\gamma$-glutamylcysteine synthetase, the rate-limiting enzyme in GSH synthesis, in HT-1080 cells, suggesting that $C A R S$ knockdown does not protect from lethality in response to inhibiting this step in glutathione synthesis (Supplementary Figure S2D). BSO, however, has been documented to induce both ferroptosis and apoptosis in different contexts, potentially complicating the interpretation of this result.

CARS knockdown inhibits erastin-induced lipid ROS formation without altering iron homeostasis. Treatment of cells with the iron chelator deferoxamine or knockdown of iron-regulating genes, such as the transferrin receptor, inhibits ferroptosis, as iron is required to induce the lipid peroxidation that is involved in the execution of ferroptosis. ${ }^{7}$
We therefore sought to determine whether CARS knockdown elicits its suppressive effects by modulating iron-regulating genes. We observed no difference in mRNA expression levels of iron-regulating genes (Supplementary Figure S3A). These data suggest that CARS knockdown does not affect iron homeostasis in cells.

Lipid ROS accumulate when GPX4 is inhibited, and this leads to the cell death phenotype that is observed in ferroptosis. GPX4, as mentioned above, can be inhibited directly with RSL3 or indirectly by depleting GSH with compounds such as erastin. We assayed for lipid ROS levels in response to erastin treatment in the context of CARS knockdown using C11-conjugated BODIPY. As expected, CARS knockdown inhibited erastin's ability to induce C11-BODIPY oxidation (Figure 3a), but did not have an effect on RSL3's ability to induce C11-BODIPY oxidation (Supplementary Figure S3B). Similar trends in intracellular ROS were observed using $\mathrm{H}_{2}$ DCFDA stain (Supplementary Figure $\mathrm{S} 3 \mathrm{C}$ ). These data suggest that CARS knockdown 
a

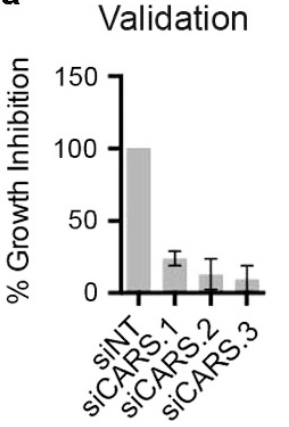

Knockdown

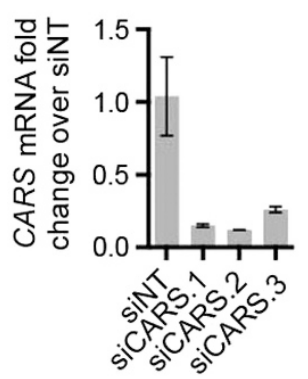

b

CARS protein

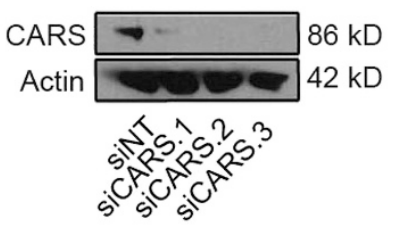

C

Effect of CARS knockdown in other cell lines
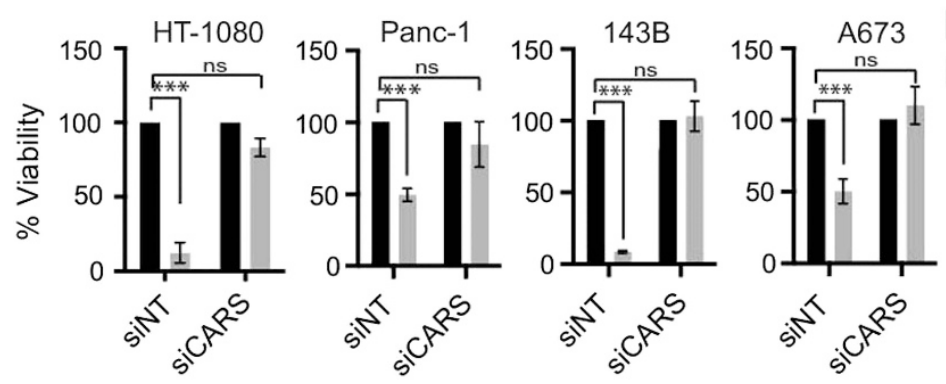

DMSO

Erastin

d CARS knockdown specifically rescues lethality induced by erastin analogs

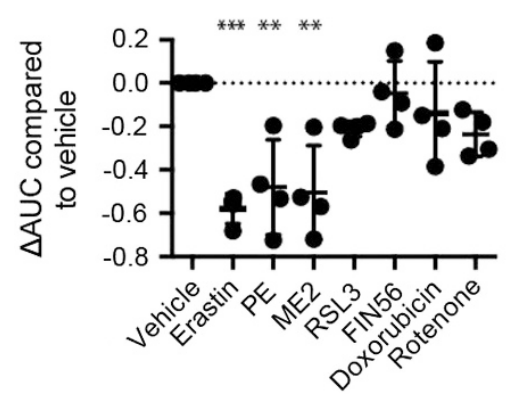

Figure 2 Validation of CARS as an inhibitor of erastin-induced ferroptosis. (a) Use of three independent siRNA sequences against CARS. Knockdown was performed in a 384-well plate for $48 \mathrm{~h}$ before the addition of $9 \mu \mathrm{M}$ erastin for $24 \mathrm{~h}$. Viability was measured with alamarBlue. mRNA levels were detected through qPCR from cells reverse transfected with siRNA in six-well format. Error bars represent one S.D. using three biological replicates. (b) CARS protein knockdown with siRNAs. (c) CARS knockdown prevents erastin lethality in a panel of cell lines. CARS (three unique siRNA sequences combined) was knocked down in 12-well format in HT-1080, Panc-1, 143B and A673 cells. After $48 \mathrm{~h}$ of knockdown, DMSO (vehicle) or $9 \mu \mathrm{M}$ erastin was added for $24 \mathrm{~h}$ before viability was measured using a ViCell (Beckman Coulter, Brea, CA, USA). Erastin-treated cell counts were normalized to DMSO-treated cell counts. Error bars represent one S.D. using three biological replicates. Two-way ANOVA, Bonferroni correction, ${ }^{* \star \star} P$-value $<0.001$ : NS, not significant relative to the indicated treatment. (d) CARS knockdown is specific for erastin-induced ferroptosis. CARS was knocked down in 6-well format and, after $48 \mathrm{~h}$, was reseeded into 384-well format. After $24 \mathrm{~h}$, lethal compounds were added in a twofold, 12-point dose curve. The highest concentrations of compound used were: erastin $(37 \mu \mathrm{M}), \mathrm{PE}(15 \mu \mathrm{M})$, ME2 $(3 \mu \mathrm{M})$, RSL3 $(10 \mu \mathrm{M})$, FIN56 $(41 \mu \mathrm{M})$, doxorubicin $(36.8 \mu \mathrm{M})$ and rotenone $(1.25 \mathrm{mM})$. AUC of each dose curve was calculated, then normalized to DMSO (vehicle) treatment, from which $\triangle \mathrm{AUC}$ was calculated and compared with DMSO. Data are from four biological replicates, one-way ANOVA compared with vehicle treatment, Bonferroni correction, ${ }^{* \star} P$-value $<0.001,{ }^{* *} P$-value $<0.01$

inhibits the ferroptotic mechanism upstream of GPX4 inhibition, but downstream of GSH depletion.

CARS knockdown rescues cells via upregulation of the transsulfuration pathway. As CARS uses cysteine in a conjugation reaction with uncharged tRNAs, we hypothesized that CARS knockdown might elevate intracellular cysteine levels, which might in turn alter intracellular glutathione levels. We performed a global metabolite analysis to detect alterations in over 300 metabolites upon CARS knockdown. There was a detectable increase in intracellular free cysteine (Figure $3 b$ ); however, there was no change in the level of reduced or oxidized intracellular GSH (Figure $3 b$ ). The most increased metabolite upon CARS knockdown was in fact cysteine-glutathione disulfide (CSSG), which would likely form in response to an increase in cysteine levels (Figure 3b). We hypothesized that CSSG could serve as a source of glutathione in times of stress, such as upon loss of cellular cysteine. Indeed, there was a slight but significant ( $t$-test, $P$-value $<0.05)$ increase even in reduced and oxidized GSH upon erastin treatment in the presence of CARS knockdown. 
We also determined from this experiment that cystathionine, a central metabolite in the transsulfuration pathway, was increased upon CARS gene knockdown (Figure 3c). We wondered whether, upon CARS knockdown, cells might become dependent upon the transsulfuration pathway to generate cysteine, leading to resistance to erastin. We examined changes in the transcriptome using RNA sequencing (using RNA-seq) to determine whether genes involved in the transsulfuration pathway may be induced upon $C A R S$ knockdown. Indeed, compared with nontargeting-siRNAtreated cells, siCARS induced genes involved in serine biosynthesis and transsulfuration (Figure $3 c$, relative abundances in Supplementary Table SI2); thus, we further investigated this pathway's involvement in leading to resistance to erastin.

In order to probe the involvement of the transsulfuration pathway in leading to resistance to erastin, we first co-treated siCARS-transfected cells with a small molecule inhibitor of the transsulfuration pathway; propargylglycine (PPG) is an inhibitor of cystathionine $\gamma$-lyase, the enzyme that converts cystathionine to cysteine in the transsulfuration pathway. ${ }^{16}$ As expected, PPG treatment on its own had no effect on erastin's lethality (Figure 3d). However, co-treatment of siCARS with PPG resensitized cells to erastin, suggesting that the transsulfuration pathway is necessary for siCARS's ability to suppress erastin's lethality. PPG co-treatment with CARS knockdown also resensitized to erastin in 143B cells (Supplementary Figure S3D). To ensure that PPG was not acting through other mechanisms known to suppress erastin, we co-treated PPG with the antioxidant $a$-tocopherol (ATOC) or with the iron chelator deferoxamine (DFOM; Supplementary Figure S3E). Neither ATOC nor DFOM's ability to suppress erastin was altered upon co-treatment with PPG, suggesting that the resensitization effect seen with $P P G$ in the presence of CARS knockdown is specific to the suppressive effect of $C A R S$, likely due to activation of the transsulfuration pathway.

We also knocked down cystathionine $\beta$-synthase $(C B S)$ simultaneously with $C A R S$ to further probe the involvement of the transsulfuration pathway in the resistance mechanism. CBS is the enzyme that converts homocysteine to cystathionine. Indeed, with simultaneous knockdown of $C B S$ and $C A R S$, inhibiting the conversion of homocysteine into cysteine, HT-1080 cells were resensitized to erastin treatment compared with cells with CARS knockdown (Supplementary Figure S3F). Use of small molecule inhibitor of CTH and genetic inhibition of CBS both resensitized CARS knockdown cells to erastin, suggesting that upregulation of the transsulfuration pathway renders cells insensitive to death induced by system $\mathrm{x}_{\mathrm{c}}^{-}$inhibition.

Induction of the integrated stress response has been shown to induce cystathionine $\gamma$-lyase to assist cells in maintaining their redox state. ${ }^{17}$ tRNA synthetase inhibition leads to phosphorylation of elF2 $a$ and activation of the transcription factor ATF4, inducing a similar transcriptional mechanism to the integrated stress response. ${ }^{18}$ CARS knockdown by siCARS induced ATF4 through phosphorylation of elF2a (Figure $3 e$ ), which may be the mechanism responsible for induction of the transsulfuration pathway.

We expected that knockdown of other tRNA synthetases would inhibit translation and induce the general amino-acid deprivation response, regardless of specific amino-acid tRNA synthetase knockdown: CARS is part of a family of tRNA synthetases. In mammals, there are tRNA synthetases for specific amino acids, with some tRNA synthetases able to charge multiple amino acids. ${ }^{19}$ We knocked down other tRNA synthetases to determine whether charging of other amino acids is essential for the suppressive effect of erastin-induced ferroptosis: methionyl-tRNA synthetase (MARS), histidyltRNA synthetase (HARS), the mitochondrial cysteinyl-tRNA synthetase (CARS2), the dual glutamyl-prolyl-tRNA synthetase (EPRS) and seryl-tRNA synthetase (SARS). Knockdown of $H A R S$ and EPRS had rescuing effects in response to erastin lethality, while MARS, SARS and CARS2 knockdown did not (Figure 3f). None of these tRNA synthetase knockdowns suppressed RSL3-induced ferroptosis (Supplementary Figure S3G). Among the other 15 tRNA synthetases, knockdown of only KARS also suppressed erastin's lethality, suggesting that the effects seen with CARS knockdown are not generalizable to all tRNA synthetases.

We studied the proliferation rate of select tRNA synthetase knockdown to see whether there was a correlation between proliferation and the cells' ability to be rescued from erastin lethality. CARS, EPRS, HARS, LARS and MARS were knocked down individually. Cell viability was measured after 24, 48, 72 and $96 \mathrm{~h}$ post tRNA synthetase knockdown. All tRNA synthetase knockdowns slowed proliferation at 72 and $96 \mathrm{~h}$ post knockdown compared with cells transfected with nontargeting siRNA (Supplementary Figure $\mathrm{S} 3 \mathrm{H}$ ), and the degree to which proliferation was affected did not correlate with their ability to rescue from erastin lethality. The specificity of each tRNA synthetase knockdown's ability to rescue from erastin's lethality could be attributed to different knockdown levels or to the cells' dependence on each tRNA synthetase. We speculated, in addition, that the effectiveness of each tRNA synthetase knockdown was due to a variably activated stress response.

To determine the basis for the difference in tRNA synthetase knockdown suppressive activity, we conducted quantitative reverse transcription-quantitative $\mathrm{PCR}$ (RT-qPCR) on mRNAs involved in the transsulfuration pathway to determine whether their upregulation was different between knockdown of different tRNA synthetases. Using MARS knockdown as a negative control and CARS, EPRS and HARS knockdown as positive controls for suppression of erastin lethality, we performed RT-qPCR on cells upon knockdown of these genes. Indeed, we found that upon EPRS knockdown, the transsulfuration pathway genes $C B S, C T H, P S A T 1$ and $P S P H$ were upregulated, whereas MARS knockdown had no effect on the mRNA levels of these genes (Figure $3 \mathrm{~g}$ and Supplementary Figure S3I). CARS and HARS knockdown upregulated $C B S$ and PSAT1. The variable response of some tRNA synthetase knockdown's ability to rescue from erastin was also observed using small molecule inhibitors of tRNA synthetases. Co-treatment with halofuginone, an inhibitor of EPRS, suppressed erastin's lethality and upregulated CBS and PSAT1, whereas histidinol, an inhibitor of HARS, did not suppress erastin and did not upregulate the transsulfuration genes (viability in Supplementary Figure S3J and mRNA in Supplementary Figure S3K). The precise mechanism by which knockdown or small molecule inhibition of some tRNA 
a

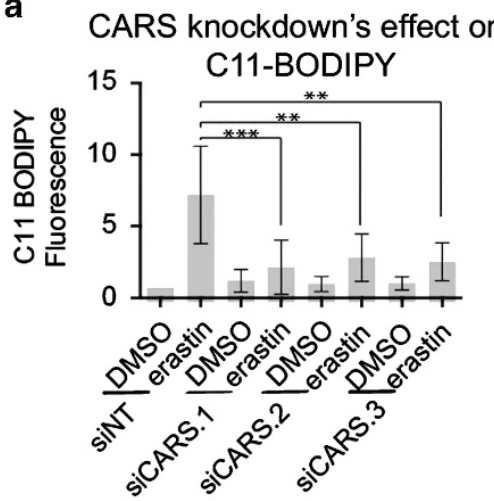

Metabolite and RNA-seq analysis in CARS knockdown

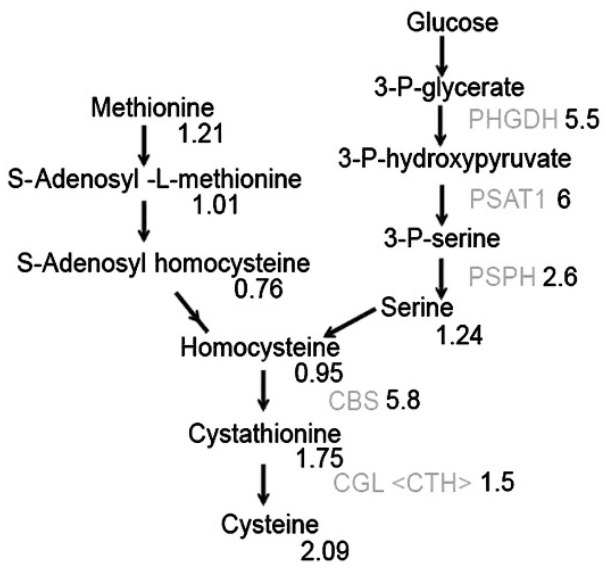

Intracellular metabolite levels
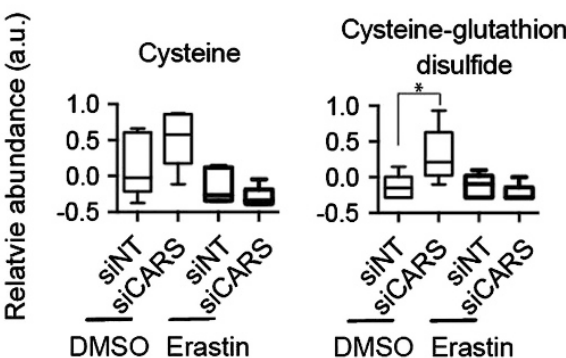

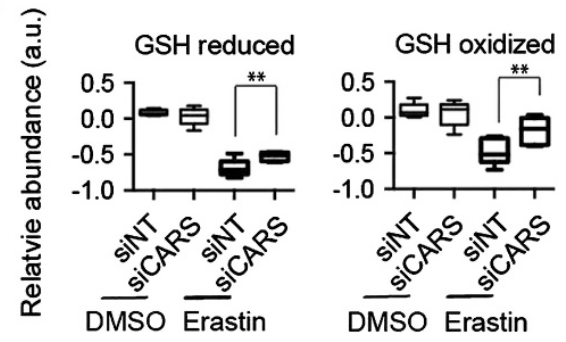

d

Propargylglycine modulation
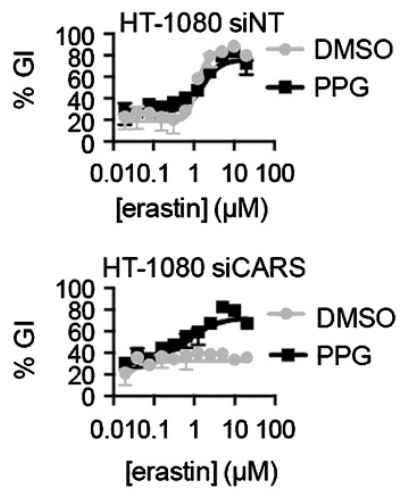

e

\section{ATF4 signaling}
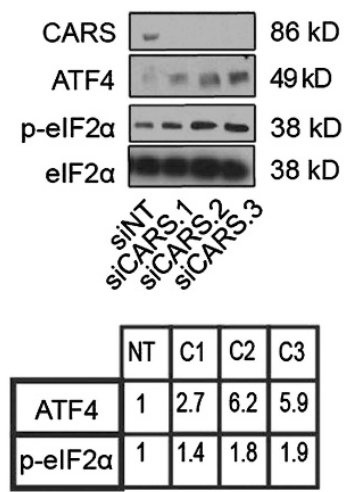

f

tRNA synthetase specificity for suppression of erastin

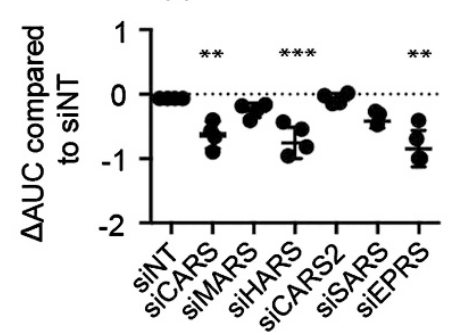

g

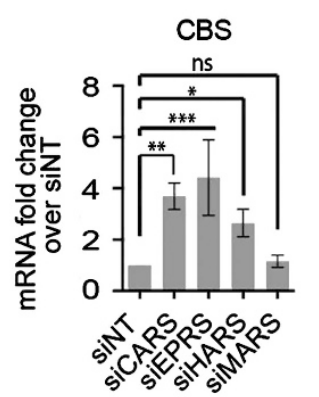

Transsulfuration and serine biosynthesis genes

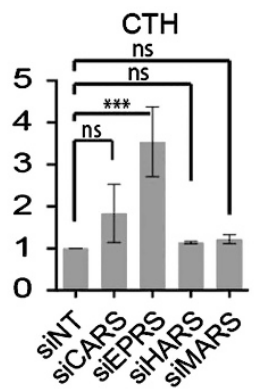

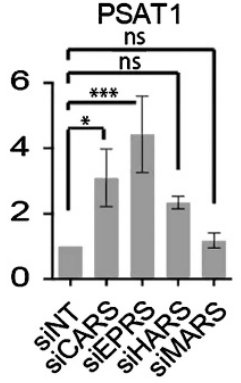

Figure 3 CARS knockdown protects from erastin-induced ferroptosis through upregulation of the transsulfuration pathway. (a) CARS knockdown inhibits lipid ROS formation in response to erastin. HT-1080 cells were reverse transfected with siRNA for $48 \mathrm{~h}$, before $9 \mu \mathrm{M}$ erastin treatment for $7 \mathrm{~h}$. C11-BODIPY was added to cells and analyzed through FACS. Two-way ANOVA, Bonferroni correction, ${ }^{* \star *} P$-value $<0.001$, ${ }^{\star *} P$-value $<0.01$. (b) Metabolite analysis of $C A R S$ knockdown cells. Intracellular cysteine, cysteineglutathione disulfide, GSH reduced and GSH oxidized are shown. Welch's $t$-test, ${ }^{* \star} P$-value $<0.05,{ }^{*} 0.05<P$-value $<0.1$. (c) Summary of metabolite (from metabolite analysis) and mRNA (from RNA-seq) levels associated with serine biosynthesis and transsulfuration. In gray are genes, followed by mRNA fold change comparing siCARS-treated cells with siNT cells. In black are metabolites, with fold change in relative metabolite levels comparing siCARS to siNT cells. Serine biosynthesis and the transsulfuration pathway are upregulated. (d) Co-treatment of CARS knockdown cells with propargylglycine resensitizes cells to erastin. HT-1080 cells were reverse transfected with siRNA for $48 \mathrm{~h}$, before reseeding into 384-well format with DMSO (vehicle), or $2 \mathrm{mM}$ propargylglycine. After $24 \mathrm{~h}$, erastin was added in a twofold, 12-point dilution series starting at $36.5 \mu \mathrm{M}$. (e) Immunoblot for proteins associated with ATF4 induction. CARS knockdown induces elF2 $\alpha$ phosphorylation and ATF4. Fold increase in proteins compared with siNT are in the table below the blots. (f) Knockdown of some tRNA synthetases prevents erastin's lethality. tRNA synthetases were knocked down in 6 -well format for $48 \mathrm{~h}$ before reseeding in 384-well format. After $24 \mathrm{~h}$, erastin was added in a twofold, 12-point dose curve, with the highest concentration of $37 \mu \mathrm{M}$ erastin. $\Delta$ AUC was calculated compared with siNTtreated cells. One-way ANOVA compared with siNT, Bonferroni correction, ${ }^{* \star} P$-value $<0.001,{ }^{* \star} P$-value $<0.05$. (g) $\mathrm{mRNA}$ levels of genes associated with serine biosynthesis and transsulfuration. siCARS, siEPRS and siHARS, all of which rescue from erastin's lethality, upregulate CBS, CTH, PSAT1 and PSPH, whereas siMARS, which does not inhibit erastin's lethality, does not induce these genes. One-way ANOVA, Bonferroni correction, ${ }^{*} P$-value $<0.05$, ${ }^{* *} P$-value $<0.01,{ }^{* * \star} P$-value $<0.001$; NS, not significant relative to the indicated treatments 

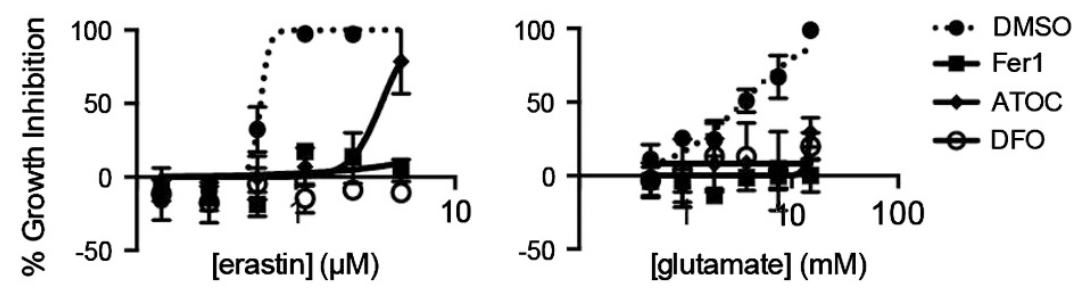

b

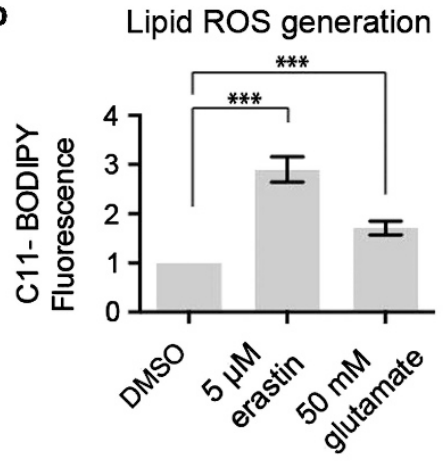

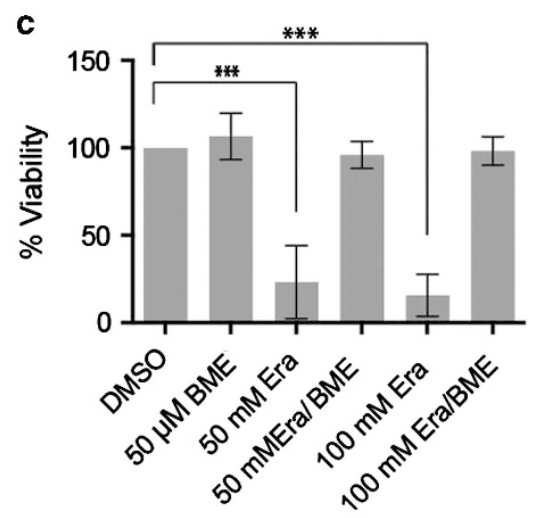

d

Knockdown of Cars in rat PC12 cells suppresses glutamate and erastin lethality
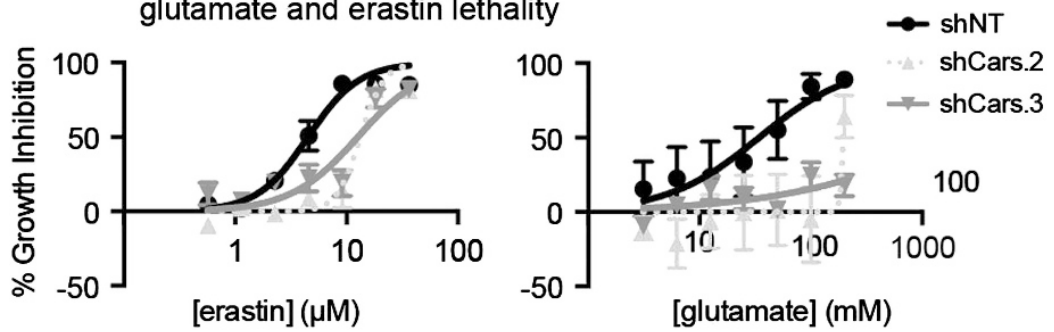

Figure 4 Cars knockdown prevents erastin-induced and glutamate-induced ferroptosis in PC12 cells. (a) Erastin and glutamate induce ferroptosis in PC12 cells. Lethal compounds were added in twofold, six-point dilution series with ferroptosis modulators for $48 \mathrm{~h}$ before viability was assessed by alamarBlue. Error bars are S.D. from three technical replicates. Data are representative of biological triplicates. (b) Erastin and glutamate induce increased C11-BODIPY staining. $t$-test, ${ }^{* \star *} P$-value $<0.01$. (c) Glutamateinduced toxicity can be rescued with $\beta$-mercaptoethanol (BME) co-treatment. PC12 cells were treated with the indicated concentration of glutamate (e) with or without $50 \mu \mathrm{M} \mathrm{BME}$ for $48 \mathrm{~h}$ before viability was assessed using a ViCell (Beckman Coulter). Error bars represent one S.D. from three biological replicates. One-way ANOVA, Bonferroni correction, ${ }^{* * \star} P$-value $<0.01$. (d) Cars knockdown using either of two shRNA sequences rescues from erastin-induced and glutamate-induced lethality in PC12 cells. Error bars represent $\mathrm{S}$. D. using technical triplicates. Data are representative of biological duplicates

synthetases leads to induction of genes associated with transsulfuration is not known. Nonetheless, upregulation of transsulfuration genes was predictive of the knockdown of tRNA synthetases to cause resistance to erastin-induced ferroptosis, suggesting that this is a key mechanism involved in their suppressive activity on ferroptosis.

CARS knockdown suppresses ferroptosis in glutamate oxidative toxicity disease model. To evaluate the potential relevance of the ability of CARS knockdown to inhibit ferroptosis to degenerative disease models, we tested the ability of CARS knockdown to prevent glutamate-induced toxicity. It has been found that glutamate can induce ferroptosis in models of glutamate toxicity, raising the question of whether CARS knockdown could prevent glutamate toxicity. ${ }^{4,12}$

To test whether CARS knockdown could prevent glutamate toxicity in a high-throughput format, we developed an assay using PC12 rat pheochromocytoma cells. PC12 cells are sensitive to glutamate-induced toxicity, which can be rescued with antioxidants. ${ }^{20}$ We first tested whether PC12 cells undergo ferroptosis in response to erastin or glutamate. PC12 cells were sensitive to both erastin and glutamate, and both lethal agents were rescued upon treatment with any of the ferroptosis inhibitors Fer-1, deferoxamine (DFO), and ATOC (Figure $4 \mathrm{a}$ ). The lethality of both erastin and glutamate was also associated with increased lipid ROS levels (Figure 4b), consistent with induction of ferroptosis. To confirm that the mechanism by which PC12 cells were dying in response to glutamate was due to cystine depletion, we co-treated cells with $\beta$-mercaptoethanol ( $\beta$-ME), which allows cells to take up cystine circumventing system $x_{c}^{-}$inhibition, and with glutamate. ${ }^{21} \beta-\mathrm{ME}$ co-treatment rescued cells from glutamate toxicity, signifying that PC12 cells undergo cell death due to cystine depletion in response to glutamate (Figure 4c). We employed shRNAs targeting rat Cars (as PC12 cells are of rat origin) and observed a suppressive effect on erastin and 
glutamate lethality (Figure 4d; mRNA, Supplementary Figure $\mathrm{S} 4 \mathrm{~A})$. These experiments suggest that inhibiting CARS in degenerative disease contexts where aberrant ferroptosis occurs may be of potential benefit, although the therapeutic index of this intervention remains to be established.

\section{Discussion}

Ferroptosis is a regulated cell death mechanism that can be induced with small molecules, and may occur in degenerative disease contexts. The death process involves lipid peroxidation; lipid peroxides are involved in diseases such as PVL, ischemia-reperfusion injury and HD. Methods to inhibit or monitor this form of cell death may be useful in these contexts.

Here we screened for genes essential for erastin-induced ferroptosis and identified CARS, a cysteinyl-tRNA synthetase. $C A R S$ knockdown inhibited lipid ROS generation upon erastin treatment, but did not have an effect on intracellular iron levels or ferroptosis induced by GPX4 inhibition. CARS is involved in cysteine metabolism, which is the rate-limiting reagent for the synthesis of glutathione, an important molecule in the regulation of the oxidative environment of cells and ferroptosis. Upon CARS knockdown, we found an increase in intracellular cysteine and also an increase in reduced and oxidized glutathione with erastin treatment, leading to maintenance of GPX4 activity and to the rescue of erastin-induced ferroptosis.

Specific tRNA synthetase knockdown, such as for CARS, HARS, EPRS and KARS, caused suppression of erastin's lethality, whereas other ARS knockdowns did not. We determined that this difference was because of each ARS knockdown's ability to induce genes associated with the transsulfuration pathway. Specifically, $C B S$, which is involved in the conversion of cystathionine to cysteine, was upregulated by ARS knockdowns that suppressed erastin's lethality. The basis for the specificity of some ARS knockdowns' ability to induce transsulfuration genes is unknown. Individual aminoacid deprivation can induce ATF4 expression to different extents. ${ }^{22}$ One possibility is that cells are more sensitive to accumulation of, or incorporation into proteins, cysteine, histidine and proline/glutamate. Another possibility is that as we were using siRNA technology to knockdown genes, knockdown of some ARSs was more efficient than others, although this was not distinguishable by RT-qPCR.

The ability of CARS knockdown to suppress ferroptosis induced by erastin, through activation of the transsulfuration pathway, explains the observation that CARS knockdown does not suppress the lethality of RSL3 or BSO. GPX4 inhibition by RSL3 cannot be rescued by increasing the pool of CSSG, GSH or cysteine, as RSL3 directly inhibits GPX4 biochemical activity. In addition, increasing the pool of intracellular cysteine would not suppress the lethality of BSO, which acts at the level of inhibiting the conjugation of cysteine to the $\gamma$-carboxyl group of glutamate. Thus, activation of the transsulfuration pathway by CARS knockdown is only expected to suppress the ferroptosis induced by cysteine depletion, caused by inhibiting the cystine-glutamate exchanger, system $x_{c}^{-}$.

GPX4 activity has been shown to be neuroprotective in HD models. ${ }^{23}$ In addition, supplementation of cysteine to mouse diets was recently shown to delay neurodegeneration in mouse models of the disease. ${ }^{24}$ The deficiency of cystathionine $\gamma$-lyase in HD models was suggested to mediate the pathophysiology of the disease. tRNA synthetase inhibitors exist as antimicrobial agents; ${ }^{25}$ however, development of a mammalian CARS inhibitor may be toxic, as tRNA synthetases are essential for protein synthesis. Mild and transient inhibition, however, may be beneficial in slowing cell death in certain physiological conditions, such as HD or ischemiareperfusion injury. Irrespective of this, the knowledge that ARS activity and the transsulfuration pathway regulate sensitivity to ferroptosis may aid in understanding and regulating this form of cell death in diverse contexts.

\section{Materials and Methods}

siRNA screening. A pooled collection of siRNAs targeting 21687 genes (Ambion V3, Grand Island, NY, USA) was screened in conjunction with the RNAi core at New York University Langone Medical Center. Three sequences targeting each gene were pooled in 384-well format and screened in duplicate. HT-1080 cells were reverse transfected using Lipofectamine RNAiMAX (Invitrogen, 13778150, Grand Island, NY, USA) with a final concentration of $9.5 \mathrm{nM}$ siRNA in each well. siRNAs targeting VDAC3 (Qiagen, SI00302155, Valencia, CA, USA) were used as a positive control on all plates. After $72 \mathrm{~h}$ of knockdown, cells were treated with $7 \mu \mathrm{M}$ erastin or DMSO. Cell viability was measured using alamarBlue after $24 \mathrm{~h}$. Growth inhibition was calculated for each erastin-treated well based on the average of the corresponding DMSO wells. The data were then analyzed using CellHTS ${ }^{2}{ }^{13}$ First, growth inhibition was normalized using NPI method (to positive and negative controls on each plate), then the mean of the duplicates were calculated before conversion to a $z$-score. The genes that rescued more than three MAD from the median against erastin's lethality based on $z$-score were rescreened in BJeLR cells using the same format as in $\mathrm{H}-1080$ cells.

Chemicals. Erastin and RSL3 were synthesized as previously described in Yang et $a l .^{6}$ and Yagoda et al. ${ }^{9}$ Doxorubicin was purchased from Tocris (Bristol, UK). Unless otherwise noted, all chemicals were purchased from Sigma-Aldrich (St. Louis, MO, USA).

Cell lines and media. HT-1080, A673 and Panc- 1 cells were obtained from the American Type Culture Collection. BJeLR cells were obtained form Robert Weinberg (Whitehead Institute). 143B cells were obtained from Eric Schon (Columbia University Medical Center). BJeLR cells were grown in DMEM (Gibco, Life Technologies Corp., Grand Island, NY, USA) with 20\% M199 (Sigma, St Louis, MO, USA), $15 \%$ heat-inactivated fetal bovine serum (FBS) and $100 \mu \mathrm{g} / \mathrm{ml}$ penicillin/ streptomycin (P/S). HT-1080 cells were grown in DMEM supplemented with $15 \%$ FBS, 1\% nonessential amino acids (Gibco) and P/S. A673, Panc-1 and 143B cells were grown in DMEM with $10 \% \mathrm{FBS}$ and P/S. All human cancer cells were grown in humidified $37^{\circ} \mathrm{C}$ with $5 \% \mathrm{CO}_{2}$. PC12 cells were purchased from American Type Culture Collection and grown in humidified $37^{\circ} \mathrm{C}, 9.5 \% \mathrm{CO}_{2}$, in DMEM (Corning, 15-013; Corning, NY, USA) supplemented with 10\% Cosmic calf serum (HyClone, $\mathrm{SH} 30087), \mathrm{P} / \mathrm{S}$ and $2 \mathrm{mM}$ glutamine.

SiRNA reverse transfection. Reverse transfection using siRNAs were performed as follows for six-well plates. siRNA was diluted in OptiMEM $(250 \mu \mathrm{l})$ to a final concentration of $10 \mathrm{nM}$ in the wells, and $250 \mu \mathrm{l}$ of OptiMEM with $2.5 \mu \mathrm{l}$ Lipofectamine RNAiMAX was added and incubated at room temperature for $30 \mathrm{~min}$. A total of 100000 cells were added to each well in regular growth media (200000 for Panc-1 cells) and knockdown was allowed to proceed for $48 \mathrm{~h}$ before reseeding for dose curves, or harvested for qPCR.

shRNA knockdown in HT-1080 cells. HT-1080 cells were infected with lentiviruses that contained shRNA expression plasmids as previously described. ${ }^{7}$ The clones used to target CARS, TRCN0000045753, TRCN0000056865, TRCN0000056866, TRCN0000045756 and TRCN0000045757, were purchased from Sigma-Aldrich.

Growth-inhibition measurements. Cells were grown in 384-well format and treated with compound for indicated times. AlamarBlue was added to each well at a final concentration of $10 \%$ for $6 \mathrm{~h}$ before reading the fluorescence (ex/em 
530/590) on a fluorescence plate reader (Victor3, Perkin Elmer, Waltham, MA, USA). For $\triangle$ AUC (area under the curve) calculations, cells were treated with letha compounds in a 12-point, twofold dilution series. The highest concentrations used were erastin $(36.6 \mu \mathrm{M})$, RSL3 $(10 \mu \mathrm{M})$, doxorubicin $(18.4 \mu \mathrm{M})$, rotenone $(25.4 \mu \mathrm{M})$, ME2 $(3 \mu \mathrm{M}), \mathrm{PE}(15.5 \mu \mathrm{M})$ and sulfasalazine $(4 \mathrm{mM})$. Cells were treated with lethal compounds for $24 \mathrm{~h}$ before the addition of alamarBlue. Growth inhibition was calculated and AUC was determined from dose-response curves. $\triangle A U C$ was calculated based on nontargeting siRNA-treated or vehicle-treated dose curves.

Lipid ROS measurements. A total of $100000 \mathrm{HT}-1080$ cells were seeded in six-well plates (Corning) after reverse transfection using $2 \mu \mathrm{l}$ Lipofectamine RNAiMAX and $10 \mathrm{nM}$ siRNA. Forty-eight hours later, cells were treated with $10 \mu \mathrm{M}$ erastin, $625 \mathrm{nM}$ RSL3 or DMSO for $8 \mathrm{~h}$. BODIPY-C11 (Invitrogen, D-3861) was added to a final concentration of $1.5 \mu \mathrm{M}$ for $20 \mathrm{~min}$ before cells were harvested by trypsinization, washed and strained through a $40 \mu \mathrm{M}$ cell strainer (BD Falcon, Franklin Lakes, NJ, USA). Cells were analyzed using a flow cytometer (Accuri C6, BD Biosciences, San Jose, CA, USA) equipped with a 488-nm laser for excitation. Data were collected from the FL1 channel, with a minimum of 10000 cells per sample. Data were analyzed as in Dixon et al. ${ }^{12} \mathrm{H}_{2}$ DCFDA (Molecular Probes, Grand Island, NY, USA) staining was done in a similar manner.

RT-qPCR. RNA was extracted using the Qiashredder and Qiagen RNeasy Mini kits (Qiagen) according to the manufacturer's protocol. A unit of $1-2 \mu \mathrm{g}$ total RNA was used for each reverse transcription reaction performed using the TaqMan RT kit (Applied Biosystems, Grand Island, NY, USA). Primer pairs were designed for target transcripts using Primer Express 3.0 (Applied Biosystems). Quantitative PCR reactions were performed using the Power SYBR Green PCR Master Mix (Applied Biosystems). Reactions were run and analyzed on a ViiA 7 (Life Technologies) qPCR instrument using absolute quantification settings. mRNA levels were normalized to ACTB internal control levels, and differences computed between samples using the $\Delta \Delta \mathrm{Ct}$ method. Primers used are in Supplementary Information.

Immunoblotting. Cells were lysed in buffer consisting of $50 \mathrm{mM}$ HEPES, $40 \mathrm{mM}$ $\mathrm{NaCl}, 2 \mathrm{nM}$ EDTA, $0.5 \%$ Triton X-100, $1.5 \mathrm{mM} \mathrm{Na}_{3} \mathrm{VO}_{4}, 50 \mathrm{mM} \mathrm{NaF}, 10 \mathrm{mM}$ sodium pyrophosphate, $10 \mathrm{mM}$ sodium beta-glycerophosphate and 1 tablet of protease inhibitor (Roche, Indianapolis, IN, USA). Cell lysates were separated on a 4-25\% Tris-gel and transferred to nitrocellulose membrane. Primary antibodies were used in the following concentrations: ATF4 (Santa Cruz, sc-200; Dallas, TX, USA) 1:100, phosphor-elF2 $\alpha$ (Cell Signaling, 3597; Danvers, MA, USA) 1: 200, elF2 $\alpha$ (Cell Signaling, 5324) $1: 1000$, CARS (AbCam, ab69454; Cambridge, MA, USA) 1:500, overnight at $4{ }^{\circ} \mathrm{C}$. Blots were incubated with 1:2000 HRP-conjugated secondary before development with SuperSignal West Pico Substrate (Pierce, 34080; Grand Island, NY, USA).

shRNA knockdown in PC12 cells. Cars was knocked down in PC12 cells using shRNA targeting rat sequences (Qiagen, KR146003P). On the day of experiment, 2 million PC12 cells were mixed with $2 \mu \mathrm{g}$ of shRNA constructs and nucelofected using Nucelofection reagent V (Lonza, VACA-1003, Basel, Switzerland). Nucelofected cells were plated in 384-well format. After $48 \mathrm{~h}$, puromycin was added to select for cells expressing the shRNA. After $24 \mathrm{~h}$, lethal compounds were added for $24 \mathrm{~h}$ before viability measurements. To confirm knockdown of Cars, nucleofected cells were seeded in six-well plate, and knockdown allowed to proceed for $48 \mathrm{~h}$, puromycin selection for $24 \mathrm{~h}$, before harvesting cells for qPCR analysis.

PC12 modulator treatment. A total of 7500 PC12 cells were seeded in 384well format with fer-1 $(1.5 \mu \mathrm{M})$, ATOC $(100 \mu \mathrm{M})$ and DFO $(152 \mu \mathrm{M})$. Lethal compounds were added in twofold, six-point dilution series, with the highest concentration used for glutamate $(20 \mathrm{mM})$ and erastin $(5 \mu \mathrm{M})$.

Statistical analysis. All experiments were done in three biological replicates unless otherwise noted. Prism 6.0b (GraphPad, La Jolla, CA, USA) was used for statistical analysis.

\section{Conflict of Interest}

The authors declare no conflict of interest.

Acknowledgements. We acknowledge the assistance of Dr. Chi Yun and Janine Recio at the NYU RNAi core for advice and assistance with the RNAi screen,
Dr. Jose Silva at Mount Sinai for reagents and assistance with LentiORF experiments and Dr. Christine Huang for help with mutagenesis experiments. The NYU RNAi core is supported by NIHA Cl 5P30CA16087-31 and NYSTEM Contract C026719. $\mathrm{MH}$ was supported by $\mathrm{NIH}$ predoctoral training grant in cancer biology (CA009503). This research was funded by the Howard Hughes Medical Institute, National Institute of Health (5R01CA097061, 5R01GM085081, R01CA161061), and New York Stem Cell Science (C026715) to BRS. BRS is an Early Career Scientist of the Howard Hughes Medical Institute.

1. Vila M, Przedborski S. Targeting programmed cell death in neurodegenerative diseases. Nat Rev Neurosci 2003; 4: 365-375.

2. Linkermann A, Skouta R, Himmerkus N, Mulay SR, Dewitz C, De Zen F et al. Synchronized renal tubular cell death involves ferroptosis. Proc Natl Acad Sci USA 2014; 111: 16836-16841.

3. Friedmann Angeli JP, Schneider M, Proneth B, Tyurina YY, Tyurin VA, Hammond VJ et al. Inactivation of the ferroptosis regulator Gpx4 triggers acute renal failure in mice. Nat Cell Biol 2014; 16: 1180-1191.

4. Skouta R, Dixon SJ, Wang J, Dunn DE, Orman M, Shimada K et al. Ferrostatins inhibit oxidative lipid damage and cell death in diverse disease models. J Am Chem Soc 2014; 136: 4551-4556.

5. Bannai S, Sato H, Ishii T, Sugita $Y$. Induction of cystine transport activity in human fibroblasts by oxygen. J Biol Chem 1989; 264: 18480-18484.

6. Yang WS, SriRamaratnam R, Welsch ME, Shimada K, Skouta R, Viswanathan VS et al. Regulation of ferroptotic cancer cell death by GPX4. Cell 2014; 156: 317-331.

7. Yang W, Stockwell B. Synthetic lethal screening identifies compounds activating irondependent, nonapoptotic cell death in oncogenic-RAS-harboring cancer cells. Chem Biol 2008; 15: 234-245.

8. Dolma S, Lessnick S, Hahn W, Stockwell B. Identification of genotype-selective antitumor agents using synthetic lethal chemical screening in engineered human tumor cells. Cancer Cell 2003; 3: 285-296.

9. Yagoda N, von Rechenberg M, Zaganjor E, Bauer A, Yang W, Fridman D et al. RAS-RAFMEK-dependent oxidative cell death involving voltage-dependent anion channels. Nature 2007; 447: 864-868.

10. Louandre C, Ezzoukhry Z, Godin C, Barbare J-C, Mazière J-C, Chauffert B et al. Iron-dependent cell death of hepatocellular carcinoma cells exposed to sorafenib. Int $J$ Cancer 2013; 133: 1732-1742.

11. Dixon SJ, Patel DN, Welsch M, Skouta R, Lee ED, Hayano M et al. Pharmacological inhibition of cystine-glutamate exchange induces endoplasmic reticulum stress and ferroptosis. eLife 2014; 3 : e02523.

12. Dixon S, Lemberg K, Lamprecht M, Skouta R, Zaitsev E, Gleason C et al. Ferroptosis: an iron-dependent form of nonapoptotic cell death. Cell 2012; 149: 1060-1072.

13. Boutros M, Bras LP, Huber W. Analysis of cell-based RNAi screens. Genome Biol 2006; 7 : R66.

14. Hahn WC, Counter CM, Lundberg AS, Beijersbergen RL, Brooks MW, Weinberg RA. Creation of human tumour cells with defined genetic elements. Nature 1999; 400: 464-468.

15. Yao P, Fox P. Aminoacyl-tRNA synthetases in medicine and disease. EMBO Mol Med 2013; 5: 332-343.

16. Abeles RH, Walsh CT. Acetylenic enzyme inactivators. Inactivation of gamma-cystathionase, in vitro and in vivo, by propargylglycine. J Am Chem Soc 1973; 95: 6124-6125.

17. Dickhout JG, Carlisle RE, Jerome DE, Mohammed-Ali Z, Jiang H, Yang $G$ et al. Integrated stress response modulates cellular redox state via induction of cystathionine gamma-lyase: cross-talk between integrated stress response and thiol metabolism. J Biol Chem 2012; 287: 7603-7614.

18. Keller TL, Zocco D, Sundrud MS, Hendrick M, Edenius M, Yum $J$ et al. Halofuginone and other febrifugine derivatives inhibit prolyl-tRNA synthetase. Nat Chem Biol 2012; 8: 311-317.

19. Guo M, Yang XL, Schimmel P. New functions of aminoacyl-tRNA synthetases beyond translation. Nat Rev Mol Cell Biol 2010; 11: 668-674.

20. Pereira CM, Oliveira CR. Glutamate toxicity on a PC12 cell line involves glutathione (GSH) depletion and oxidative stress. Free Radic Biol Med 1997; 23: 637-647.

21. Ishii T, Bannai S, Sugita Y. Mechanism of growth stimulation of L1210 cells by 2-mercaptoethanol in vitro. Role of the mixed disulfide of 2-mercaptoethanol and cysteine. J Biol Chem 1981; 256: 12387-12392.

22. Palii SS, Kays CE, Deval C, Bruhat A, Fafournoux P, Kilberg MS. Specificity of amino acid regulated gene expression: analysis of genes subjected to either complete or single amino acid deprivation. Amino Acids 2009; 37: 79-88.

23. Mason RP, Casu M, Butler N, Breda C, Campesan S, Clapp J et al. Glutathione peroxidase activity is neuroprotective in models of Huntington's disease. Nat Genet 2013; 45: 1249-1254.

24. Paul BD, Sbodio JI, Xu R, Vandiver MS, Cha JY, Snowman AM et al. Cystathionine gamma-lyase deficiency mediates neurodegeneration in Huntington's disease. Nature 2014; 509: 96-100.

25. Hurdle JG, O'Neill AJ, Chopra I. Prospects for aminoacyl-tRNA synthetase inhibitors as new antimicrobial agents. Antimicrob Agents Chemother 2005; 49: 4821-4833. 